\title{
Campylobacter jejuni enters gut epithelial cells and impairs intestinal barrier function through cleavage of occludin by serine protease $\mathrm{Htr} A$
}

\author{
Aileen Harrer ${ }^{1}$, Roland Bücker ${ }^{2}$, Manja Boehm ${ }^{1}$, Urszula Zarzecka ${ }^{1,4}$, Nicole Tegtmeyer ${ }^{1}$, Heinrich Sticht ${ }^{3}$, \\ Jörg D. Schulzke ${ }^{2}$ and Steffen Backert ${ }^{1 *}$ (1)
}

\begin{abstract}
Campylobacter jejuni secretes HtrA (high temperature requirement protein A), a serine protease that is involved in virulence. Here, we investigated the interaction of HtrA with the host protein occludin, a tight junction strand component. Immunofluorescence studies demonstrated that infection of polarized intestinal Caco-2 cells with C. jejuni strain 81-176 resulted in a redistribution of occludin away from the tight junctions into the cytoplasm, an effect that was also observed in human biopsies during acute campylobacteriosis. Occludin knockout Caco-2 cells were generated by CRISPR/Cas9 technology. Inactivation of this gene affected the polarization of the cells in monolayers and transepithelial electrical resistance (TER) was reduced, compared to wild-type Caco-2 cells. Although tight junctions were still being formed, occludin deficiency resulted in a slight decrease of the tight junction plaque protein ZO-1, which was redistributed off the tight junction into the lateral plasma membrane. Adherence of C. jejuni to Caco-2 cell monolayers was similar between the occludin knockout compared to wild-type cells, but invasion was enhanced, indicating that deletion of occludin allowed larger numbers of bacteria to pass the tight junctions and to reach basal membranes to target the fibronectin receptor followed by cell entry. Finally, we discovered that purified C. jejuni HtrA cleaves recombinant occludin in vitro to release a $37 \mathrm{kDa}$ carboxy-terminal fragment. The same cleavage fragment was observed in Western blots upon infection of polarized Caco-2 cells with wild-type C. jejuni, but not with isogenic $\triangle$ htrA mutants. HtrA cleavage was mapped to the second extracellular loop of occludin, and a putative cleavage site was identified. In conclusion, HtrA functions as a secreted protease targeting the tight junctions, which enables the bacteria by cleaving occludin and subcellular redistribution of other tight junction proteins to transmigrate using a paracellular mechanism and subsequently invade epithelial cells.
\end{abstract}

Keywords: Occludin, Tight junction, E-cadherin, Campylobacter, Protease, HtrA

\section{Introduction}

Campylobacter jejuni are Gram-negative, motile bacteria with a spirally shaped body that commensally colonize the intestines of birds and mammals. However, in humans C. jejuni causes gastroenteric infections, and as such $C$. jejuni is among the most common causes of

\footnotetext{
*Correspondence: Steffen.Backert@fau.de

${ }^{1}$ Division of Microbiology, Dept. of Biology, University of Erlangen-

Nuremberg, Staudtstr. 5, 91058 Erlangen, Germany

Full list of author information is available at the end of the article
}

zoonotic illnesses worldwide. Infections are frequently caused by contaminated chicken meat and other animalderived products. Infected individuals may sporadically develop secondary diseases such as Guillian-Barré or Miller-Fisher syndrome that are more serious than the usually self-limiting diarrhea in campylobacteriosis [13]. Upon reaching the gut, a first step in the pathogenic process leading to tissue damage is invasion of the bacteria into epithelial cells, as was demonstrated in biopsies of infected patients and by the use of in vitro infection 
assays $[2,4]$. For this process, $C$. jejuni uses several outer membrane proteins to adhere to and invade into the cells, for instance CadF and FlpA, which bind to the extracellular matrix protein fibronectin followed by cell entry in an integrin-dependent fashion [5-9]. Interestingly, fibronectin and integrins are predominantly located on the basal side of enterocytes, but how $C$. jejuni reaches these basal receptors for a long time remained unknown. Paracellular transmigration of the pathogen is an intriguing possibility, and recently a protein that could be involved in this process was identified as the serine protease HtrA $[10,11]$.

Many bacteria contain one or more HtrA homologs [12-18]. HtrA proteins combine both protease and chaperone functions and are commonly located in the periplasmic space. Various HtrAs are composed of an amino-terminal signal peptide, a trypsin-like serine protease domain and one or two PDZ-domains responsible for protein-protein interaction [19, 20]. HtrA of Escherichia coli is the best studied model, and this species contains three homologs called DegP, DegQ and DegS. Their main function is to protect $E$. coli against heat and other stresses, and to remove misfolded proteins [19, 21, 22]. C. jejuni contains only one HtrA homolog, and this periplasmic protein can be secreted into the extracellular space, where it is able to cleave the extracellular domain of the adherens junction protein E-cadherin [10]. This helps C. jejuni to transmigrate between neighbouring cells to reach the basal side the polarized epithelium, a process that depends on HtrA activity $[11,23]$.

The question addressed here is how $C$. jejuni acts on tight junctions, which are located above the adherens junctions facing to the gut lumen and tighten the lateral intercellular space (LIS) to form a barrier against the intestinal lumen. Tight junctions are composed of a protein network localized at the apical site of epithelial and endothelial cell layers. Their so-called "fence" function maintains the cell's polarity, while their "gate" function depends on openings, which only allow small molecules to pass the apical-basal barrier [24, 25]. Tight junction strands are formed by several proteins including tricellulin, occludin, claudins and junction adhesion molecules (JAMs) [25-27]. All these proteins interact with the tight junction plaque proteins like $\mathrm{ZO}-1, \mathrm{ZO}-2$ and $\mathrm{ZO}-3$ or cingulin, which are linked to the intracellular actin cytoskeleton. The first strand-forming tight junction protein identified was occludin, which forms homodimers in the cellular membrane. It contains four transmembrane domains at the $\mathrm{N}$-terminus forming two extracellular loops that participate in the tight junction and a long intracellular C-terminal tail. The first extracellular loop is rich in glycine and tyrosine residues [28], whereas the second loop contains two conserved cysteine residues that are important for homodimerisation [29]. The exact function of occludin is still unclear, but it has been suggested to regulate tight junction assembly and the distribution of other tight junction proteins within the tight junction strand meshwork [30-33].

The impact of $C$. jejuni on intestinal tight junctions is widely unknown, but it was shown that the bacteria can co-localize with occludin during infection of the E12 cell line [34]. Infection with C. jejuni alters the localization of occludin within the host cell membrane, leading to a re-distribution of the protein, but the involved molecular process remained unclear $[35,36]$. In addition, treatment of T84 cells with enriched outer membrane vesicles (OMVs) derived from $C$. jejuni was associated with the cleavage of host cell proteins $[37,38]$. Since HtrA can be incorporated as cargo into released OMVs or secreted as soluble protein, we investigated whether tight junction proteins are targeted by the protease activity of $C$. jejuni $\mathrm{HtrA}$. The results presented here suggest that occludin is a direct cleavage target of $C$. jejuni $\mathrm{HtrA}$, which results in opening of tight junctions followed by paracellular transmigration of the bacteria.

\section{Materials and methods Campylobacter strains}

Campylobacter jejuni strain 81-176 was used in this study, together with an isogenic knockout mutant 81-176 4 trA and the complemented mutant 81-176 AhtrA/htrA (Table 1), [11, 39]. All C. jejuni strains were grown on Campylobacter blood-free selective Agar Base containing Campylobacter growth supplement (Oxoid, Wesel, Germany) or on Mueller-Hinton (MH) agar plates amended with $30 \mu \mathrm{g} / \mathrm{mL}$ kanamycin or $20 \mu \mathrm{g} /$ $\mathrm{mL}$ chloramphenicol at $37{ }^{\circ} \mathrm{C}$ under microaerobic conditions generated by CampyGen gas packs (Oxoid) for $48 \mathrm{~h}$.

\section{Caco- 2 cell cultures, infection assay and immunofluorescence staining}

Caco-2 cells (ATCC HTB-37) were cultured in 6-well or 12-well plates with DMEM medium containing $4 \mathrm{mM}$ glutamine (Invitrogen, Karlsruhe, Germany) and 10\% FCS (Invitrogen). All C. jejuni strains grown on Campylobacter blood-free selective Agar Base plates were resuspended in $\mathrm{BHI}$ to desired optical densities $\left(\mathrm{OD}_{600} \mathrm{~nm}\right)$ followed by infection of Caco-2 cells with a multiplicity of infection (MOI) of 100. After infection for $24 \mathrm{~h}$, the cells were washed with PBS and immunofluorescence staining was performed as described [40]. Briefly, cells were fixed with $4 \%$ paraformaldehyde (PFA) at room temperature for $10 \mathrm{~min}$ followed by permeabilization with $0.25 \%$ Triton-X100 for $1 \mathrm{~min}$ and blocking with 5\% BSA in PBS for $1 \mathrm{~h}$. Proteins were stained with $\alpha$-occludin antibodies, recognizing the $\mathrm{C}$-terminus of the protein (Thermo 
Table 1 Bacterial strains and plasmids

\begin{tabular}{|c|c|c|}
\hline Strain/plasmid & Genotype & References \\
\hline C. jejuni 81-176 & Wild-type & [11] \\
\hline C. jejuni 81-176 $\Delta$ htrA & C. jejuni strain 81-176 with $\triangle$ htrA deletion, $\mathrm{Cat}^{\mathrm{R}}$ & [11] \\
\hline C. jejuni 81-176 $\triangle$ htrA/htrA & $\Delta h t r A$ deletion complemented with wild-type $h t r A$ from C. jejuni 81-176, $\operatorname{Kan}^{R}$ & [39] \\
\hline E. coli BL21(DE3)pLysS & 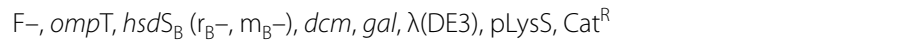 & Promega \\
\hline pKB1004 & pET28a, wt htrA from the C. jejuni NCTC11168 strain, C-terminal 6x His tag, Kan $^{R}$ & [69] \\
\hline pUZCj4 & pET26b, wt htrA from the C. jejuni NCTC11168 strain, C-terminal $6 \times$ His tag, $\mathrm{Kan}^{R}$ & This work \\
\hline
\end{tabular}

Fisher Scientific, Darmstadt, Germany, cat. nr. 42-2400) or $\alpha$-Campylobacter antibodies (Dako, Glostrup, Denmark). Nuclei were stained using 4'-6-diamidino2-phenylindole dihydrochloride (DAPI) (Thermo Fisher Scientific). As secondary antibodies FITC (fluorescein isothiocyanate)-conjugated goat $\alpha$-rabbit and TRITC (tetramethylrhodamine isothiocynate)-conjugated goat $\alpha$-rabbit (Thermo Fisher Scientific) were used. Samples were analysed using a Leica SP5 confocal fluorescence microscope and different lasers (Leica Microsystems, Wetzlar, Germany), performed at the OICE (FAU Erlangen-Nuremberg, Germany). Images were obtained via LAS AF computer software (Leica Microsystems) and optimized in brightness and contrast with ImageJ-win64 (version 2.0).

\section{Colon biopsies and confocal laser-scanning microscopy}

Biopsies were obtained from patients who underwent routine colonoscopy due to acute diarrhea or for preventive examination of colon cancer. The biopsies were taken from the sigmoid colon as previously described [41]. The described symptoms were mainly watery diarrhea associated with abdominal cramps and stool frequencies of 10-20 times within $24 \mathrm{~h}$. The diagnosis of infection by $C$. jejuni was approved by stool culture. The C. jejuni-positive patients were in the acute phase of infection between 3 and 7 days after the first symptoms appeared and revealed elevated $\mathrm{C}$-reactive protein (CRP) levels ranging from 20 to $150 \mathrm{mg} / \mathrm{L}$ and boosted blood leukocyte counts of $11.7 \times 10^{9} / \mathrm{L}$ to $16.7 \times 10^{9} / \mathrm{L}$ [41] . The inflammatory parameters in the $C$. jejuni-negative control group were normal (data not shown). All individuals had been informed of the risks and gave written consent. The biopsies were directly fixed in Tissue-Tek (Sakura, Alphen a/d Rijn, The Netherlands) in liquid nitrogen, stored at $-80{ }^{\circ} \mathrm{C}$ and then cryo-sectioned for immunofluorescence staining. Primary antibodies recognizing human tight junction proteins, rabbit $\alpha-\mathrm{ZO}-1$ (\# 61-7300) and mouse $\alpha$-occludin (\# 33-1500), were used for detection (Invitrogen, Carlsbad, CA, USA) as well as $\alpha$-Campylobacter (Santa Cruz Biotechnology Inc., Santa
Cruz, CA, USA, \# sc-58101) at 1:100 dilutions. Secondary AlexaFluor antibodies from goat $\alpha$-mouse or $\alpha$-rabbit IgG (Invitrogen) were used at 1:500 dilution. The tight junction proteins $\mathrm{ZO}-1$ and occludin were visualized by confocal laser-scanning microscopy (Zeiss LSM510, Jena, Germany) and analyzed by Zeiss Image Examiner software.

\section{Inactivation of occludin in Caco-2 cells by CRISPR/Cas9}

The occludin gene was inactivated in Caco-2 cells by application of the CRISPR/Cas9 technology. For this purpose, two plasmids were obtained from Santa Cruz (Heidelberg, Germany), one of which contained the 20 nucleotide gRNA for a region in the occludin gene [Occludin CRISPR/Cas9 KO Plasmid (human), sc-418271] and the HDR-plasmid [Occludin HDRPlasmid (human), sc-418271-HDR] that was used for stable transfection. The procedure of mutagenesis was performed using a protocol provided by the manufacturer.

\section{Cloning, expression and purification of HtrA C. jejuni}

The $h t r A$ gene of $C$. jejuni strain 11168 was introduced into NcoI and XhoI restriction sites of the pET26b expression plasmid, giving rise to vector pUZCj4. The E. coli BL21(DE3)pLysS strain transformed with pUZCj4 was used to overproduce wild-type HtrA with the C-terminal $6 \times$ His tags of the pET System (Novagen, San Diego, CA, USA). The bacteria were grown at $37^{\circ} \mathrm{C}$ in Luria-Bertani (LB) broth supplemented with kanamycin $(50 \mu \mathrm{g} / \mathrm{mL})$ to OD of 0.8. Next, the HtrA expression was induced by addition of $0.5 \mathrm{mM}$ isopropyl- $\beta$-D-thiogalactopyranoside (IPTG). After induction overnight, the temperature of the bacterial culture was reduced to $30{ }^{\circ} \mathrm{C}$. The bacteria were centrifuged $(10 \mathrm{~min}, 5000 \times \mathrm{g})$ and the pellet was resuspended in $15 \mathrm{~mL}$ of the lysis buffer BH10 (50 mM HEPES pH 8.0, $300 \mathrm{mM} \mathrm{KCl,} 10 \mathrm{mM}$ imidazole $\mathrm{pH}$ 8.0). Afterwards, the cells were lysed by addition $1 \mathrm{mg} / \mathrm{mL}$ lysozyme followed by sonication. Next, to remove DNA from lysates, DNase I ( $5 \mu \mathrm{g} / \mathrm{mL})$ was used. Lysates were cleared by centrifugation $25,000 \times g$ for $30 \mathrm{~min}$ at $4{ }^{\circ} \mathrm{C}$. 
The nickel-affinity chromatography (Ni-NTA, Qiagen, Germany) under native conditions was applied to purify the protein as described by Zarzecka and co-workers [42]. Briefly, non-specifically bound proteins were washed from the loaded resin with buffer BH40 $(50 \mathrm{mM}$ HEPES pH 8.0, $300 \mathrm{mM} \mathrm{KCl}, 40 \mathrm{mM}$ imidazole $\mathrm{pH} 8.0$ ), and HtrA was eluted with buffer BH200 (50 mM HEPES pH 8.0, $300 \mathrm{mM} \mathrm{KCl}, 200 \mathrm{mM}$ imidazole $\mathrm{pH}$ 8.0). The purity of the proteins was estimated to be more than $95 \%$ as judged by SDS-PAGE electrophoresis.

\section{Immunoblotting}

Proteins were resolved in 12\% SDS-PAGE gels and blotted as described [43]. Prior to antibody incubation, the membranes were blocked in TBST buffer $(140 \mathrm{mM} \mathrm{NaCl}$, $25 \mathrm{mM}$ Tris- $\mathrm{HCl} \mathrm{pH} \mathrm{7.4,0.1 \%} \mathrm{Tween-20)} \mathrm{with} \mathrm{3 \%} \mathrm{BSA}$ or $5 \%$ skim milk for $1 \mathrm{~h}$ at room temperature. The first antibody, rabbit $\alpha$-occludin $(\mathrm{H}-279$, sc-5562), mouse $\alpha$-occludin (E5) (sc-133256, Santa Cruz), mouse $\alpha-\beta$ Actin (Sigma Aldrich, Taufkrichen, Germany), or rabbit $\alpha$-HtrA (courtesy of Lone Brøndsted, [44]) as indicated, was incubated overnight at $4{ }^{\circ} \mathrm{C}$. As secondary antibody, horseradish peroxidase-conjugated $\alpha$-rabbit or polyvalent $\alpha$-mouse immunoglobulin was used (Life Technologies, Darmstadt, Germany). Antibody detection was performed with the ECL Plus chemiluminescence Western Blot kit (GE Healthcare Life Sciences, Munich, Germany) [45].

\section{Transwell assay}

Caco-2 cells or the corresponding occludin knockout cells were grown for 14 days to confluent monolayers in a transwell filter system as described and the transepithelial electrical resistance (TER) was determined every 2 days $[11,39]$.

\section{Gentamicin protection assay}

Caco-2 cells and the occludin knockout cells were infected with C. jejuni 81-176, $\Delta h t r A$ and $\Delta h t r A / h t r A$ at an MOI of 100. After infection for $6 \mathrm{~h}$, the cells were washed three times with $1 \mathrm{~mL}$ of pre-warmed DMEM medium per well to remove non-adherent bacteria. To determine the colony forming units (CFU) corresponding to intracellular bacteria only, extracellular bacteria were killed by incubation with $250 \mu \mathrm{g} / \mathrm{mL}$ gentamicin (Sigma Aldrich) at $37^{\circ} \mathrm{C}$ for $2 \mathrm{~h}$, after which the cells were washed three times with medium [46]. Cells were then lysed with $1 \mathrm{~mL}$ of $0.1 \%(\mathrm{w} / \mathrm{v})$ saponin (Sigma Aldrich) in PBS at $37^{\circ} \mathrm{C}$ for $15 \mathrm{~min}$ and resuspended, diluted, and plated on $\mathrm{MH}$ agar plates. To determine the total CFU corresponding to cell-associated bacteria (intra- and extracellular combined), following the three wash steps the infected monolayers were directly incubated with $1 \mathrm{~mL}$ of $0.1 \%(\mathrm{w} / \mathrm{v})$ saponin in $\mathrm{PBS}$ at $37{ }^{\circ} \mathrm{C}$ for $15 \mathrm{~min}$ without prior treatment with gentamicin [47]. All experiments were performed in triplicates.

\section{In vitro cleavage of recombinant occludin}

Three $\mu$ g of recombinant GST-tagged human occludin (antikoerper-online.de) was incubated with $1 \mu \mathrm{g}$ of purified C. jejuni HtrA in $25 \mu \mathrm{L}$ HEPES buffer $(50 \mathrm{mM}, \mathrm{pH}$ 7.4) for $16 \mathrm{~h}$ at $37^{\circ} \mathrm{C}$. To remove LPS, aliquots of $200 \mu \mathrm{g} /$ $\mathrm{mL} \mathrm{HtrA}$ were incubated with $20 \mu \mathrm{g} / \mathrm{mL}$ of polymyxin B (Sigma Aldrich) for $1 \mathrm{~h}$ at room temperature as described [48] and did not change the activity of HtrA and outcome of the experiments. The resulting cleavage products were analysed by immunoblotting stained with either $\alpha$-occludin $\mathrm{H}-279$ or with rabbit $\alpha$-GST antibodies (GE Healthcare, Freiburg, Germany).

\section{Bioinformatics}

The secondary structure in the vicinity of the cleavage site was assessed using PSIPRED [49]. Potential HtrA cleavage sites were detected by a pattern-based search using PATTINPROT (https://npsa-prabi.ibcp.fr/cgi-bin/ npsa_automat.pl?page=/NPSA/npsa_pattinprot.html). The search pattern [VITA]-[VITA]-x(2,4)-[DN] was derived from an inspection of $H$. pylori HtrA cleavage sites in E-cadherin [50].

\section{Statistics}

All data were evaluated via two-tailed Mann-Whitney test with GraphPad Prism 6 (Version 6.01). The obtained p-values $p \leq 0.05{ }^{(*)}, p<0.01^{(* *)}, p<0.0001^{(* * * * *)}$ ) were defined as statistically significant.

\section{Results \\ Redistribution of occludin in Caco-2 cells during C. jejuni infection depends on HtrA}

The effect of $C$. jejuni HtrA on the localization of occludin in the tight junctions was studied by immunofluorescence staining of in vitro infected cells. Confluent grown monolayer of polarized Caco- 2 cells were infected with wild-type strain $81-176$, its isogenic $\Delta h t r A$ knockout mutant, or $\Delta h t r A$ complemented with wild-type $h \operatorname{trA}(\Delta h \operatorname{tr} A / h t r A)$ as control. After infection for $24 \mathrm{~h}$, the cells were fixed and stained for immunofluorescence visualization of occludin (green) and C. jejuni (red). This demonstrated that proper tight junctions with belt-like pattern of occludin are formed in the non-infected control (Fig. 1a, inlay, blue arrows), while infection with wild-type or complemented $C$. jejuni interrupted the typical occludin distribution (Fig. 1b, d, white arrows). Interestingly, this effect was not seen when the htrA gene was 
deleted (Fig. 1c). Thus, infection of polarized Caco-2 cells with bacteria containing an intact $h \operatorname{tr} A$ gene altered the localization of occludin (Fig. 1b, d). These observations demonstrate that HtrA is involved in the disturbance and relocalization of occludin during infection. However, co-localization of occludin with the bacteria was not observed.

\section{Tight junction protein distribution in biopsies} from campylobacteriosis patients

To confirm that the in vitro observations had relevance to $C$. jejuni infection of humans in vivo, the subcellular distribution of tight junction proteins was investigated by confocal laser-scanning microscopy of three human colon biopsies obtained from acutely $C$. jejuni infected patients and from healthy controls, respectively. The

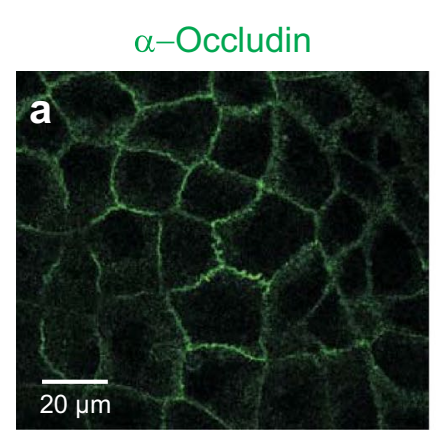

$\alpha-C . j e j u n i$
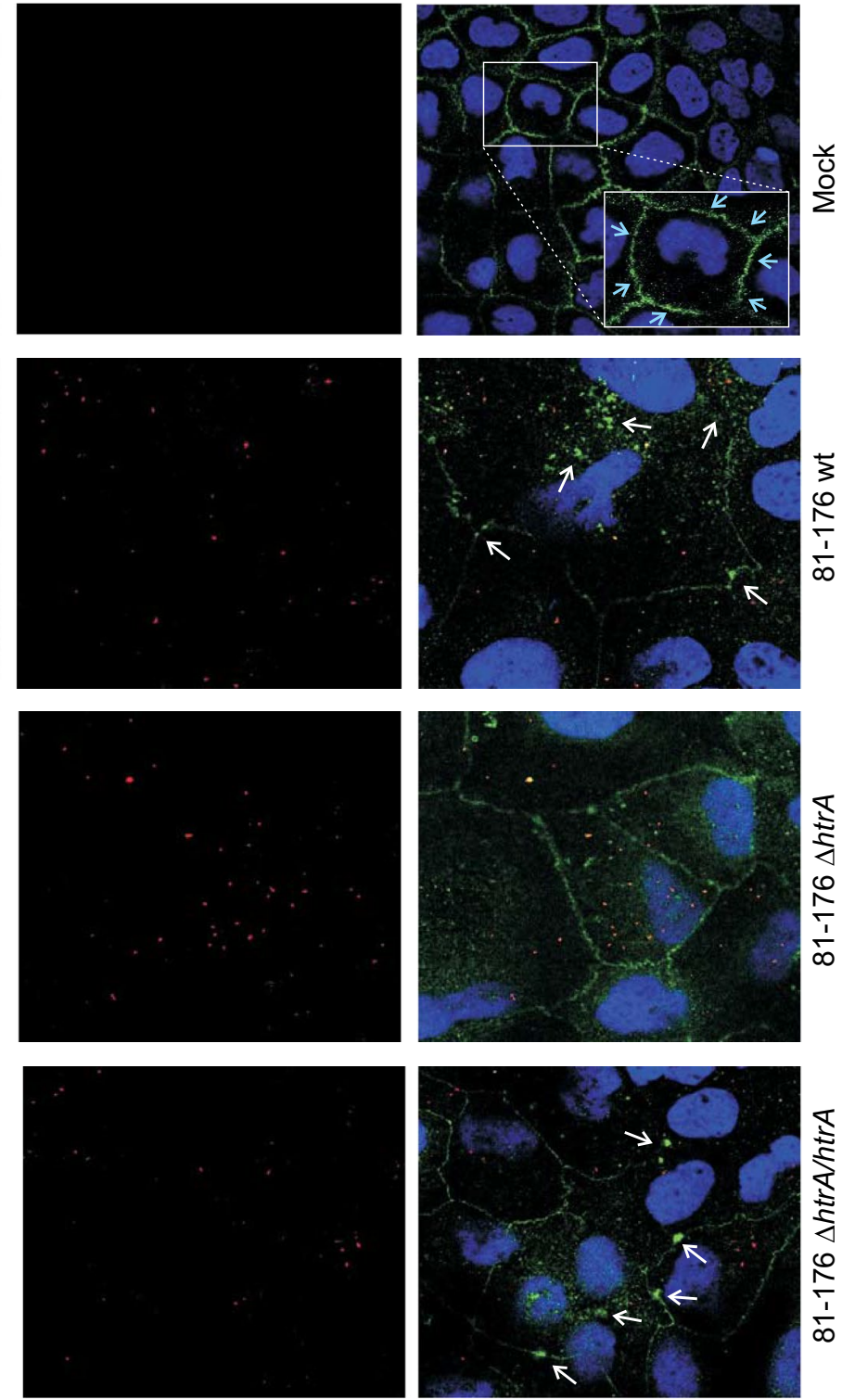

Fig. 1 Infection of Caco-2 cells with C. jejuni disturbs the occludin patterns in a htrA-dependent manner. a-Occludin (green) and a-C. jejuni immunostaining of Caco-2 mock control cells (a) or cells infected with wild-type (wt) strain 81-176 (b), $\triangle$ htrA knockout mutant (c) or $\triangle$ htrA complemented with wild-type $h t r A$ (d). The inlay in panel A shows the belt-like pattern of occludin around the polarized cells as expected (blue arrows). Infection was performed for $12 \mathrm{~h}$ at an $\mathrm{MOI}$ of 100. The pictures revealed a redistribution of upon wt infection but not $\triangle$ htrA mutant (white arrows). Co-localization of occludin with C. jejuni was not observed. DAPI staining (blue) was used for visualization of the DNA in the nuclei 
absence or presence of $C$. jejuni in all samples was confirmed by antibody staining as well as stool culture as described [41]. In biopsies from non-infected controls, overview images showed that occludin was co-localizing with zonula occludens protein-1 (ZO-1) within the tight junctions as expected (Fig. 2a, b). In contrast, mucosal biopsies obtained from patients infected with $C$. jejuni revealed the redistribution of occludin and ZO-1 (Fig. 2c, $\mathrm{d}$, white arrows). Close inspection of enlarged sections showed that occludin and ZO-1 changed their localization from predominantly apical sites in the controls (Fig. 3a, blue arrows) and became visible intracellularly after infection (Fig. 3b, white arrows). These data confirm that occludin and also other tight junction proteins like ZO-1 are relocated during acute $C$. jejuni infection.

\section{Knockout of occludin in Caco-2 cells weakens the tight junctions}

In order to investigate the role of occludin relocalization by $C$. jejuni further, we inactivated the corresponding gene in Caco- 2 cells by CRISPR/Cas9 mutagenesis. Absence of occludin expression in three knockout cell clones was confirmed by Western blotting using a monoclonal $\alpha$-occludin antibody (Fig. 4a). The $\alpha$ - $\beta$-actin control blot demonstrated that equal protein was loaded on the gel. Phase contrast microscopy revealed only slight phenotypical differences between the confluent cell monolayers (Fig. 4b). To assess whether inactivation of occludin in Caco-2 cells introduced any changes in the tight junctions, the cells were stained for ZO-1 protein. This identified that inactivation of occludin caused a slight decrease of ZO-1 assembly in tight junctions, while the amount of ZO-1 in the cytoplasm increased (Fig. 4c). In addition, the functionality of the tight junctions was investigated by determining the transepithelial electrical resistance (TER). For this purpose, Caco-2 wild-type and occludin knockout mutant cells were grown for 13 days in a Transwell filter system to reach a confluent monolayer, and TER was measured every 2 days. The overall TER values increased during this period, however, significantly lower values were obtained for the knockout compared to wild-type cells (Fig. 5a-c). Thus, inactivation of occludin in Caco-2 cells leads to a slight destabilization, but not complete disruption of the tight junctions.

\section{Adhesion and invasion of $C$. jejuni depends on expression of occludin}

Next, adhesion and invasion of $C$. jejuni upon infection of confluent wild-type Caco-2 and occludin knockout cells was determined by the gentamicin protection assay (Fig. 6). We observed that the $\Delta h t r A$ deletion mutant adhered to and invaded into either cell line at significant lower levels compared to wild-type bacteria (Fig. 6a, b).
In addition, the binding and invasion capacity of each strain was slightly higher for the occludin knockout cells compared to wild-type Caco-2, and reached statistical significance for the $\Delta h t r A$ mutant. These results suggest that disturbance of occludin in polarized Caco-2 cells can enhance the adhesion and invasion capabilities of $C$. jejuni, probably as a result of weaker and more penetrable tight junctions.

\section{HtrA cleaves occludin upon C. jejuni infection of Caco-2 cells}

The above results suggest that $C$. jejuni HtrA has an impact on the function of occludin. To test if $C$. jejuni $\mathrm{HtrA}$ is involved in cleaving occludin, confluent Caco-2 monolayers were infected with wild-type and $\Delta h \operatorname{tr} A$ mutant $C$. jejuni for $24 \mathrm{~h}$ followed by immunoblotting using the polyclonal $\alpha$-occludin $(\mathrm{H}-279)$ as well as $\alpha-\mathrm{HtrA}$ and $\alpha-\mathrm{GAPDH}$ antibodies as controls (Fig. 7a). The results show that besides a band of full-length occludin at $65 \mathrm{kDa}$ and two isoforms $(\sim 45-50 \mathrm{kDa}$, asterisk), an additional band appeared at $37 \mathrm{kDa}$. Since the $\alpha$-occludin $\mathrm{H}-279$ antibodies are directed against the $\mathrm{C}$-terminus of the protein, we proposed that the $\mathrm{C}$-terminus of occludin is cleaved off upon infection. As control, probing of the blot with the above mentioned monoclonal $\alpha$-occludin antibody did not detect the cleaved $37 \mathrm{kDa}$ fragment, because the recognized epitope lies in the N-terminus of the protein (data not shown). Remarkably, this cleavage was dependent on HtrA, as the $37 \mathrm{kDa}$ product was predominantly visible when cells had been infected with wild-type $C$. jejuni compared to the $\Delta h t r A$ deletion mutant (Fig. 7a).

\section{In vitro cleavage assay and identification of a HtrA cleavage site in occludin}

To confirm that the $37 \mathrm{kDa}$ product of occludin is indeed cleaved by HtrA, we performed in vitro cleavage assays using recombinant proteins. For this purpose, N-terminally GST-tagged human occludin (monomers of $\sim 100 \mathrm{kDa}$ and dimers of $\sim 200 \mathrm{kDa}$ ) was incubated with purified HtrA. After $16 \mathrm{~h}$ of incubation, the in vitro cleavage reactions were subjected to immunoblotting using $\alpha$-occludin (H-279) and $\alpha$-GST antibodies (Fig. 7b). The blot showed the appearance of the same sized C-terminal $37 \mathrm{kDa}$ fragment of occludin through cleavage by $\mathrm{HtrA}$. The remaining $63 \mathrm{kDa} \mathrm{N}$-terminal fragment was also visible after staining with $\alpha$-GST (Fig. 7b). Mapping of these cleavage products suggests that HtrA can cleave occludin in the second extracellular loop of the protein (Fig. 7c, top). Inspection of the occludin sequence in the second extracellular loop (amino acids 196-243) indeed identified the presence of a protease cleavage site corresponding to A/TGLYVD (Fig. 7c, bottom), which shares 


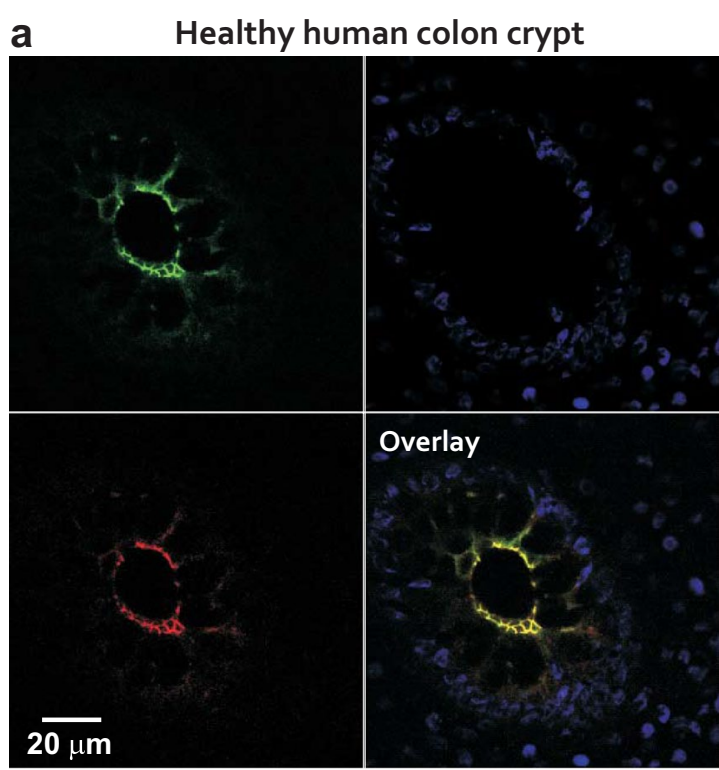

C

C. jejuni infected

b
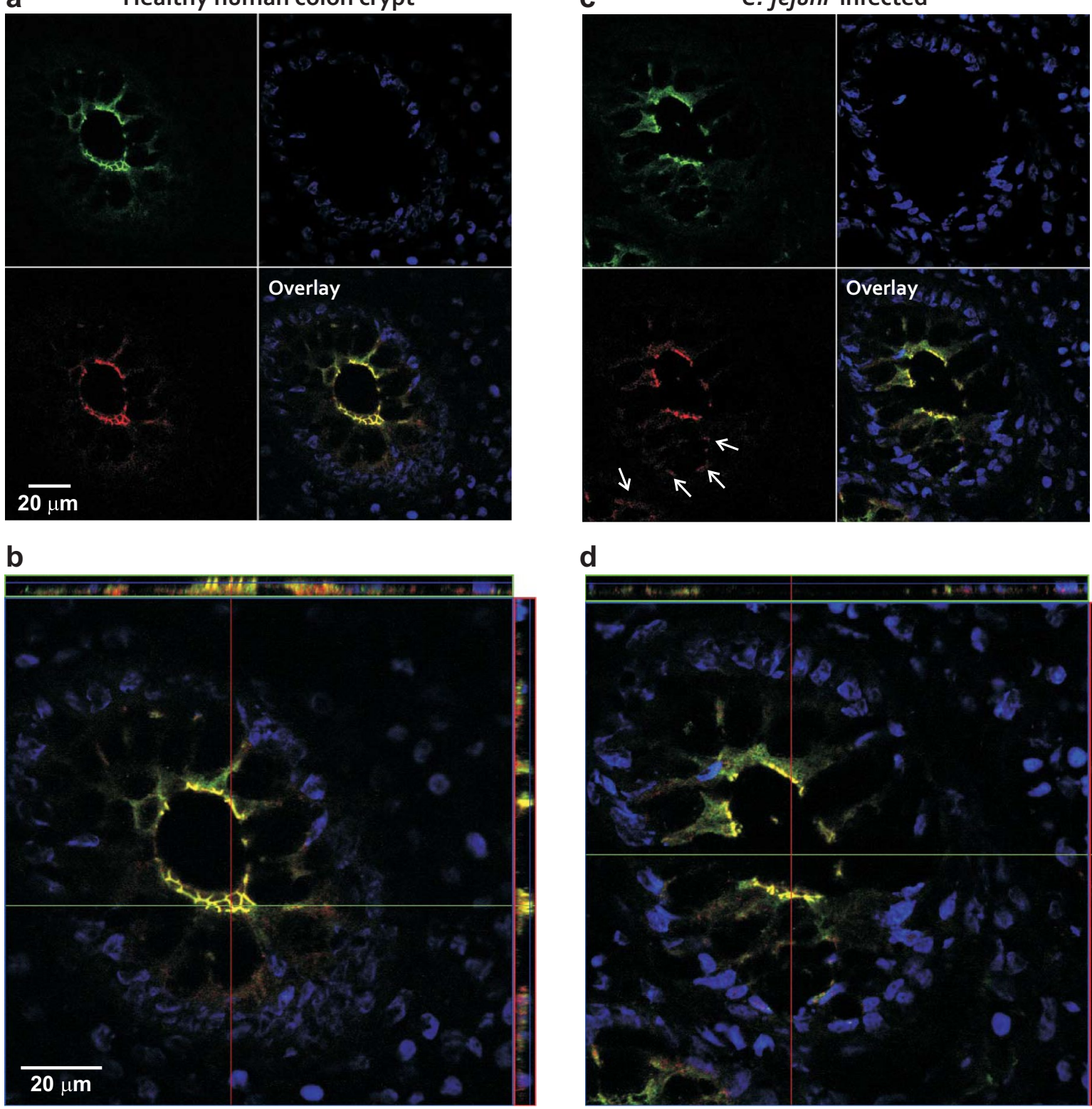

d

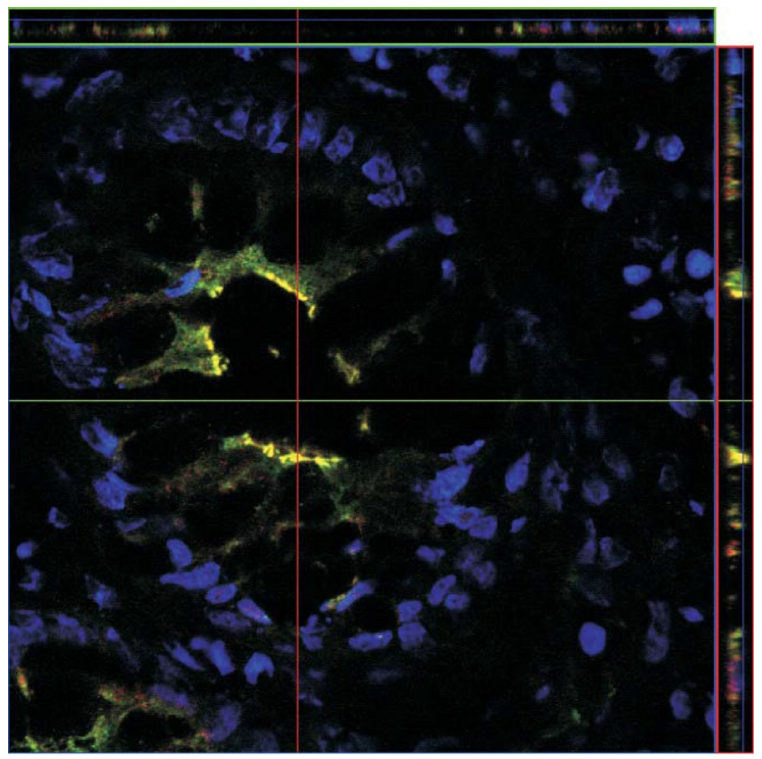

\section{Occludin ZO-1 DAPI}

Fig. 2 Tight junction protein distribution in intestinal biopsies from healthy individuals and campylobacteriosis cases. Confocal laser-scanning microscopy of human colon crypts from a non-infected individual $(\mathbf{a}, \mathbf{b})$ and an acute $C$. jejuni infection $(\mathbf{c}, \mathbf{d})$. The images show clockwise immunostaining for zonula occludens protein-1 (ZO-1, green), occludin (red), nuclei by use of DAPI staining (blue) and a merged image in which co-localization of the tight junction proteins ZO-1 and occludin appears as yellow. White arrows indicate redistributed occludin signals in the infected biopsies

significant resemblance to the signature motif [VITA][VITA]-x-x-D-[DN] defined previously for preferential H. pylori HtrA cleavage sites in E-cadherin [50]. We also predicted the secondary structure of the loop to be in close vicinity of the cleavage site. This analysis indicates the presence of short elements of secondary structure flanking the cleavage site, whereas the site itself is located in a random coil region suggesting a high accessibility for cleavage by HtrA (Fig. 7d). 

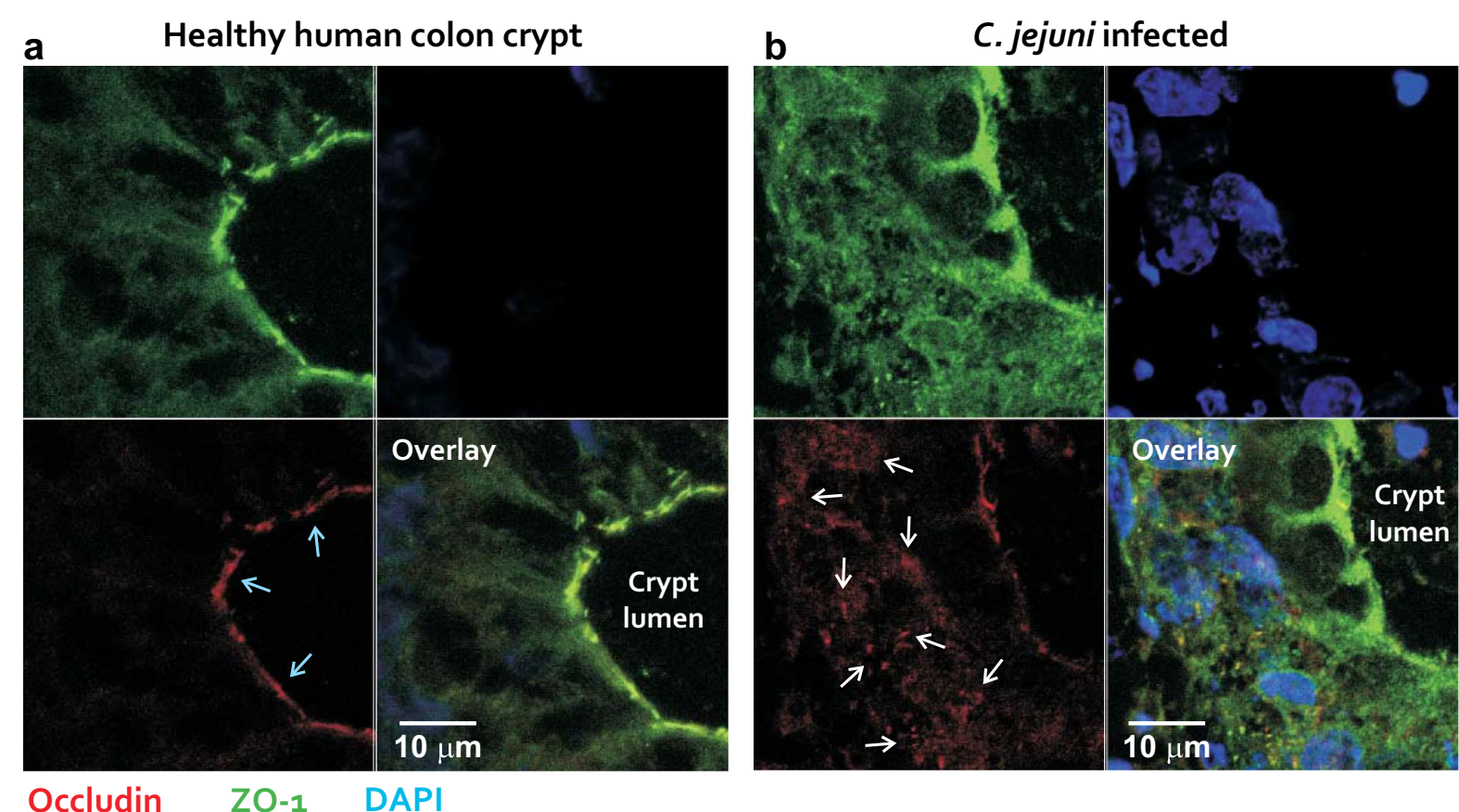

Fig. 3 Enlarged images of gut epithelial cells from biopsies of non-infected and C. jejuni-infected patients. Confocal laser-scanning microscopy images at high magnification show in detail colon crypts from a healthy patient (a) and patient infected with C. jejuni (b). The biopsies are stained against occludin (red), ZO-1 (green) and the DNA with DAPI (blue). The overlay images (bottom, right) show the co-localization from occludin and ZO-1 (yellow) in the non-infected control (a) and in a disturbed tight junction pattern in the infected sample (b). Blue arrows indicate the apical labelling of occludin in non-infected controls, while white arrows indicate cytosolic occludin signals in the infected samples

\section{Discussion}

The tight junction complex is a key regulator of epithelial cell functions. Besides providing a selective barrier to the lumen, tight junction proteins have been shown to be involved in differentiation, cell-to-cell adhesion and proliferation of healthy epithelial cells [51]. In addition, tight junctions are an important target for microbial pathogens that manipulate the host, in order to support their own survival, spread and sometimes persistence. Out of a myriad of potential molecular targets, some bacterial and viral pathogens have selected a subset of proteins in the apical-junctional complex of epithelial cells [52]. For instance, Clostridium perfringens produces virulence factors that directly interact with tight junction proteins [26]. Eichner and co-workers [53] demonstrated that C. perfringens enterotoxin is able to destroy the epithelial cells, for which the toxin initially binds to tight junction proteins, using claudin- 4 as a receptor. Another example is Vibrio cholerae, which produces a metalloprotease, haemagglutinin/protease (HA/P), which functions as a cytotoxin. In fact, $\mathrm{HA} / \mathrm{P}$ degrades occludin, thereby affecting ZO-1. Since ZO-1 is bound directly to occludin, these events can induce a signaling pathway influencing the host cell actin cytoskeleton [54]. However, the cleavage site of HA/P in occludin has not yet been identified.
More information is available for secreted HtrA produced by the gastric pathogen Helicobacter pylori that cleaves E-cadherin at various positions, and the cleavage sites have been recently identified by mass spectrometry and Edman degradation, respectively [50].

The most important result of the currently presented work is that HtrA of C. jejuni can cleave occludin in vitro, during infection of cultured polarised Caco-2 cells and in biopsies of campylobacteriosis cases in vivo, which affects its distribution in epithelial cells and various bacteria-host cell interactions. Recently, Elmi and co-workers [38] demonstrated in in vitro experiments that purified OMVs of $C$. jejuni are able to trigger the cleavage of E-cadherin and occludin, however, whether this cleavage is caused by a bacterial protease or activated human protease remained unclear. Here, we provide evidence that $C$. jejuni HtrA cleaves occludin directly. Our previous analysis of the cleavage sites in E-cadherin employed by $H$. pylori HtrA revealed that they are flanked by hydrophobic amino acids, preferably valine, isoleucine, threonine and alanine [50]. A consensus cleavage sequence has been defined to occur at the [VITA]-[VITA]- $x-x-D-[D N]$ motif [50]. However, not all HtrA cleavage sites strictly confer this pattern and there is a certain degree of 
a

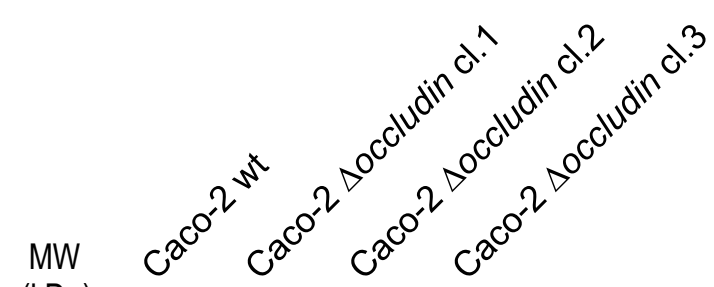

$(\mathrm{kD})$
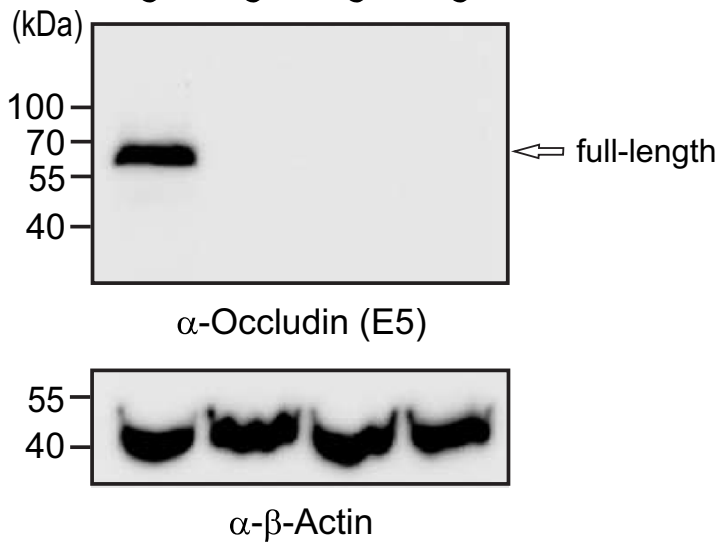

b

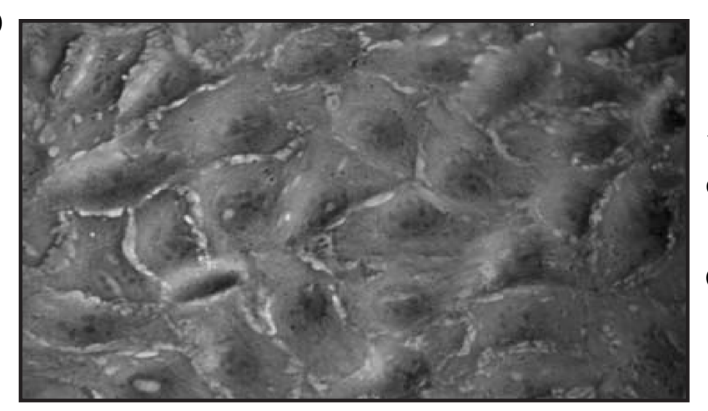

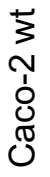

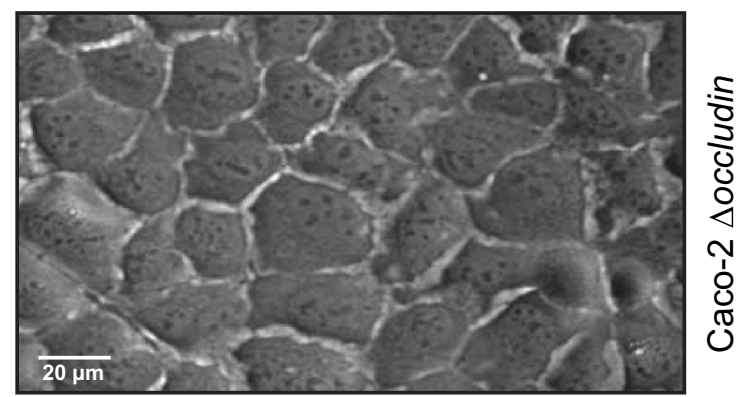

C
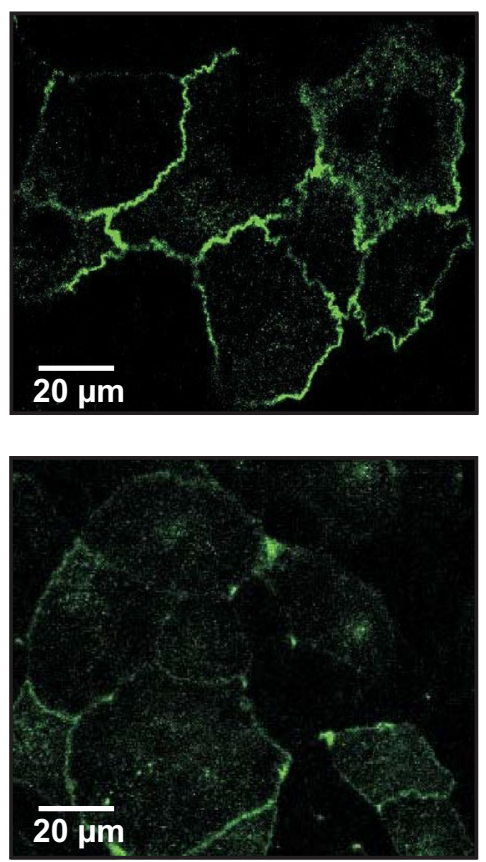

KO-Plasmid
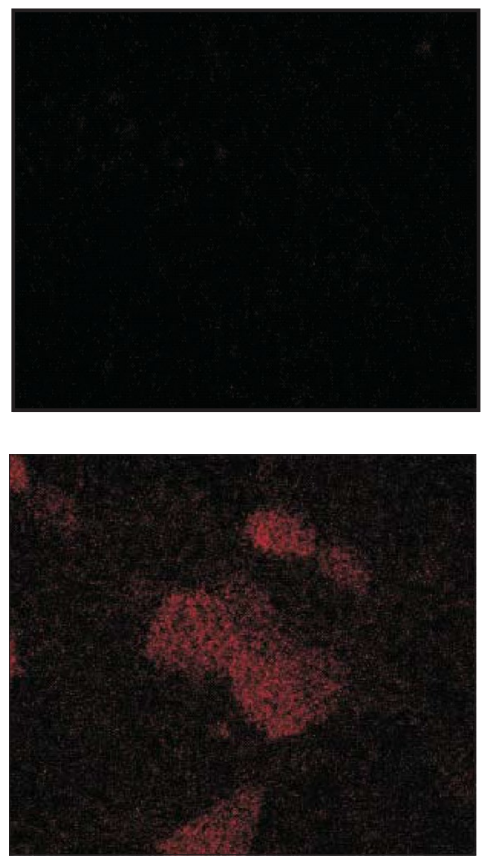

DAPI/overlay

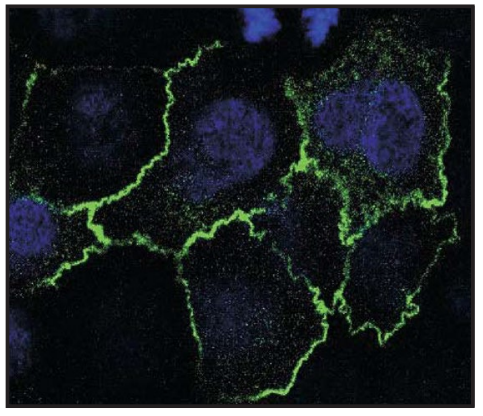

药

N

ర্

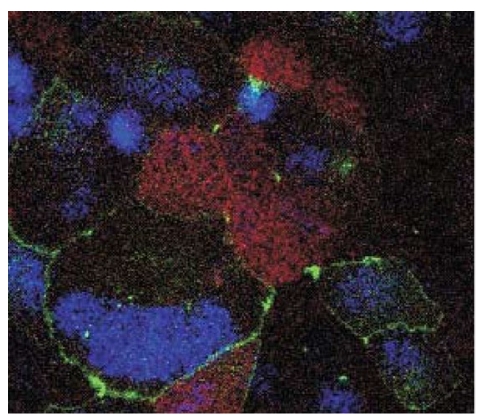

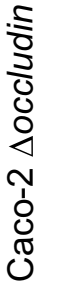

Fig. 4 CRISPR/Cas9 knockout of occludin in Caco-2 cells reveals partial downregulation of ZO-1. Three individual occludin knockout clones of Caco-2 cells were analysed. Western blotting confirms the presence of occludin in Caco-2 wild-type (wt) cells and absence in the knockout cells (a). Phase contrast microscopy of Caco-2 wt cells (top) and occludin knockout cells (bottom) revealed only slight phenotypic differences (b). Immunofluorescence staining of Caco-2 wt (top) and occludin knockout cells (bottom) stained for ZO-1 (green), presence of the HDR-plasmid (red) and DNA in the nuclei by DAPI (blue). The red fluorescence derives from the CRISPR-Cas9 plasmid, which was used for stable transfection (c)

variability concerning the conservation and spacing of the $\mathrm{C}$-terminal acidic residue [50]. To take this observation into account, we defined the more fuzzy pattern
[VITA]-[VITA]-x(2,4)-[DN] for the identification of a HtrA cleavage site in occludin. The fact that only one site in the second extracellular loop matches this 


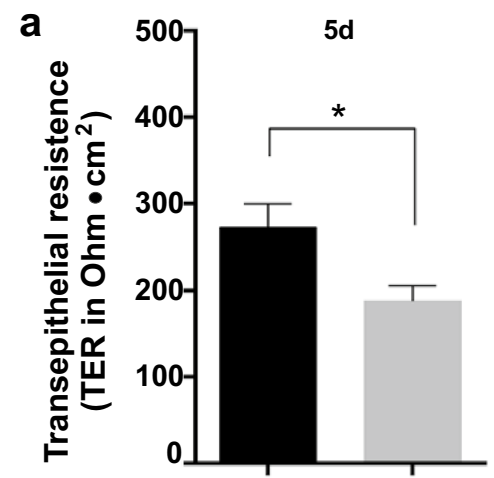

b

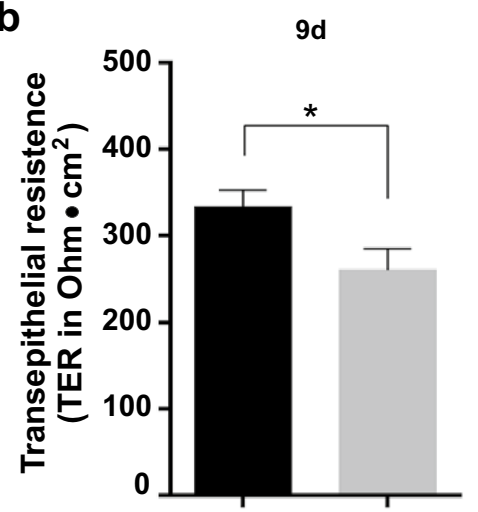

C

$13 d$

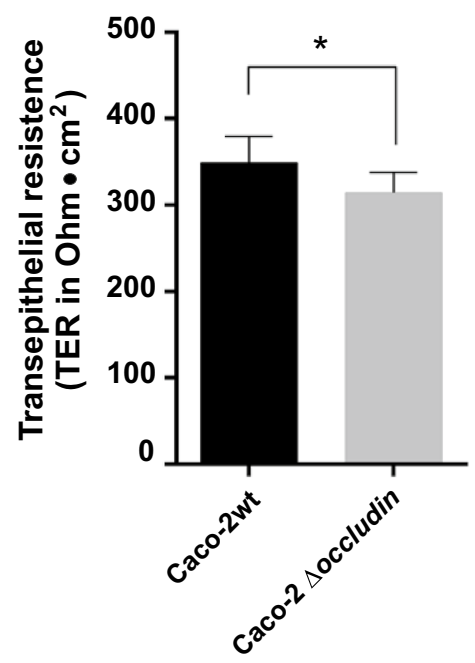

Fig. 5 Transepithelial resistance (TER) in Caco-2 wild-type (wt) and occludin knockout during cell differentiation monitored over 2 weeks. The TER measurements revealed increasing TER values both in the wt and knockout Caco-2 cells over 5 days (a), 9 days (b) and 13 days (c). However, the values in the occludin knockout cells were always slightly below wt levels, indicating a significant permeability defect in the knockout. $n=15, p<0.05\left(^{*}\right)$, Mann-Whitney test. Data represented mean \pm SEM

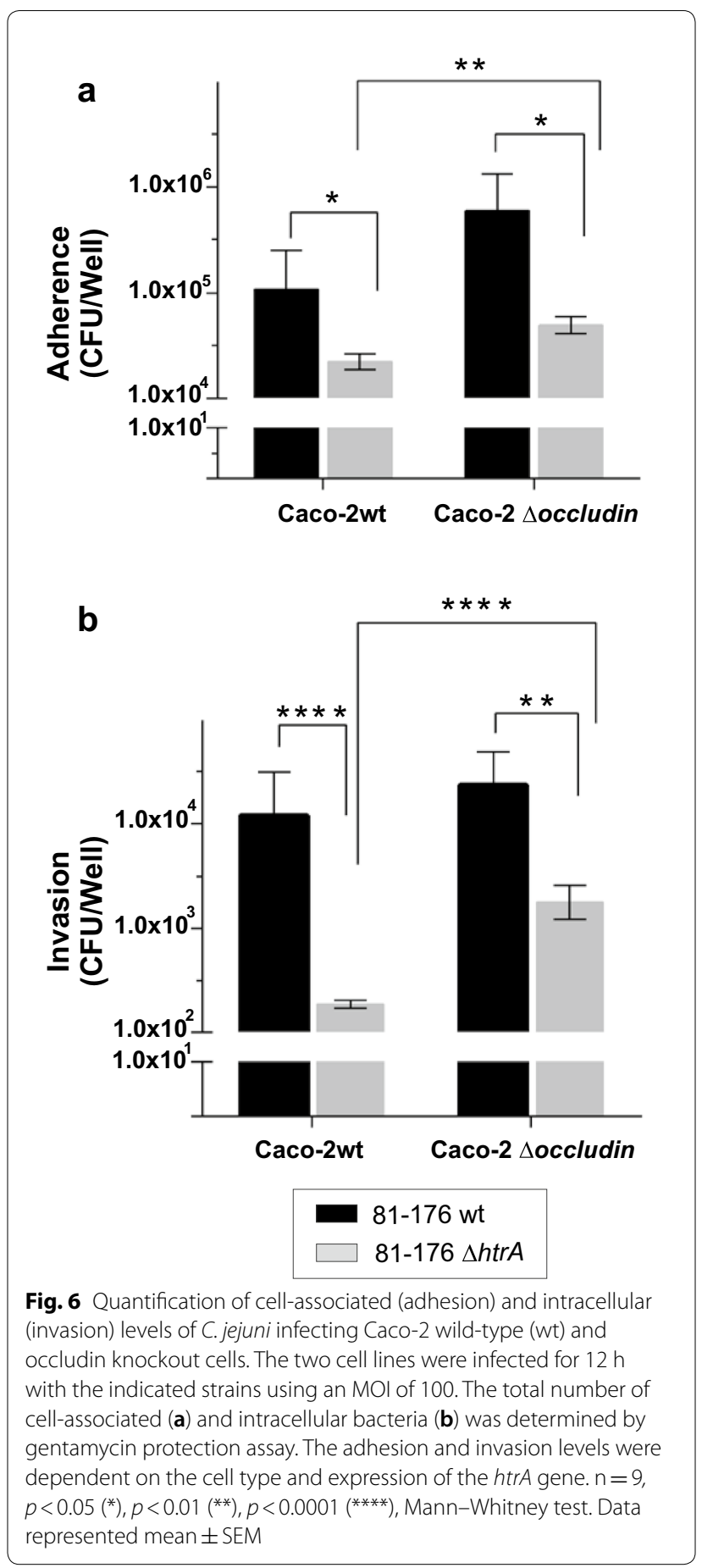

pattern and that this site is characterized by lack of secondary structure, renders it a perfect candidate for recognition and cleavage by HtrA. Interestingly, the size of the obtained $37 \mathrm{kDa} C$-terminal cleavage product is identical to that produced by $H$. pylori HtrA [55], which is associated with the relocation of occludin from tight junctions into the cytoplasm seen in another study [56], 
suggesting that both HtrAs may use the same cleavage site in occludin, which was identified here. However, a faint band of $37 \mathrm{kDa}$ was also present in cell lysates upon infection with $C$. jejuni $\Delta h t r A$. We propose that it could derive from cleavage by another yet unknown secreted C. jejuni protease or by a host cell protease that maybe activated upon infection. One such candidate is Meprin-A, a metalloprotease, which is highly expressed at the luminal interface of the intestine and can cleave a variety of substrates in vitro, including occludin [57]. Another candidate is metalloprotease-9 (MMP-9). It was shown that activation and cellular export of MMP-9 led to the cleavage of occludin during infection by hepatitis $C$ virus [58]. If Meprin-A and/ or MMP-9 can be activated by $C$. jejuni is not clear and should be investigated in future studies.

Occludin contains two extracellular loops, which are involved in tight junction protein interactions and are important to maintain the cellular barrier function. In mice, it has been shown that inactivation of the occludin gene had only a slight effect on the barrier function of tight junctions for ions, as characterized by impedance spectroscopy, and thus did not result in pronounced gut leakage $[59,60]$. The cell shape or growth behaviour of the epithelial knockout cells remained unchanged, and tight junctions were still being formed, suggesting that occludin may not essential for tight junction assembly. However, it is debated whether in a knockout animal other tight junction proteins can eventually functionally compensate the lack of occludin, at least in part [59, 60]. Nevertheless, instant effects on occludin cleavage by Campylobacter HtrA might cause a (temporary) barrier defect. Also, occludin deficiency has been discussed in the context of an increased macromolecule passage through epithelia, especially since occludin does influence the distribution of tricellulin (occludin presence drives tricellulin into the tricellular tight junction), a tight junction protein with important tightening function for the tricellular tight junction, at a site in the epithelium, where three or four cells meet [61].

These observations are in line with our experiments using Caco-2 cells and corresponding occludin knockout cells produced by CRISPR/Cas9 technology, showing that occludin is not absolutely essential for the formation of tight junctions. Our results suggest that there are downstream effects of occludin inactivation, as we clearly observed a decrease in presence of membrane-associated ZO-1. The polarization capacity of the knockout cell lines was followed over time by TER determination. This showed that inactivation of occludin resulted in slightly lower TER values compared to wild-type Caco- 2 cells. From this, we conclude that polarization of the cells might have been decreased to some degree, and although tight junctions were still being formed, their function seems to be slightly impaired when occludin is inactivated. It appears that these cells are able to partly compensate the lack of occludin by restructuring other tight junction proteins, which may explain why levels of ZO-1 were slightly lower in our Caco-2 occludin knockout cells [36].

The C-terminal cytoplasmic tail of occludin comprises a coiled-coil domain, which is able to trigger homodimerization of the protein and binding to ZO-1 [62-64]. Previous work has also shown that disruption of epithelial barrier functions can be achieved by transfecting occludin mutant constructs lacking the intracellular tail [65], suggesting that the coiled-coil domain is functionally important for maintaining the cellular tight junction barrier [66]. Interestingly, immunoblotting of native and SDS-PAGE separated cell extracts revealed occludin dimers and monomers simultaneously on gels. The dimer is presumably an antiparallel intermolecular interaction by disulfide bond formation via cysteine residue 409 in human occludin [67]. It is concluded that the redox-dependent dimerization of occludin may play a regulatory role in the tight junction assembly under physiological and pathological conditions. We also have

\footnotetext{
(See figure on next page.)

Fig. $7 \mathrm{HtrA}$ cleaves occludin during C. jejuni infection in vivo and in cleavage assays in vitro. a Immunoblotting of protein extracts from polarized Caco-2 cell monolayers infected with C. jejuni wild-type (wt) or $\triangle$ htrA mutant. The blots were stained with polyclonal a-occludin antibodies recognizing the $\mathrm{C}$-terminus of the protein. The $\mathrm{a}-\mathrm{H}$ trA and a-GAPDH blots served as controls. Besides the full-length protein ( $65 \mathrm{kDa})$, a cleaved C-terminal fragment $(37 \mathrm{kDa}$ ) was visualized with a-occludin antibodies upon infection with wt bacteria. The asterisk marks a double band at $\sim 45-50 \mathrm{kDa}$, presumably corresponding to two isoforms of occludin recognized by this antibody. $\mathbf{b}$ Recombinant GST-tagged human occludin was incubated with purified $\mathrm{HtrA}$ in in vitro cleavage assays. Cleavage of full-length GST-occludin (monomer at $100 \mathrm{kDa}$ ) resulted in the production of the same $37 \mathrm{kDa}$ C-terminal fragment as detected upon infection. Reprobing of the blot with a-GST antibodies resulted in the detection of the corresponding $\mathrm{N}$-terminal cleavage fragment of $63 \mathrm{kDa}$. The asterisks mark the position of GST-occludin dimers migrating at $200 \mathrm{kDa}$. c Mapping of the occludin cleavage fragments. An HtrA cleavage site was localized in the second extracellular loop between the transmembrane (TM) domains 3 and 4 as indicated. Bioinformatical investigation revealed the exact cleavage position as indicated on the bottom. $\mathbf{d}$ Secondary structure prediction for the second extracellular loop. The predicted type of secondary structure (Helix ' $H^{\prime}$, Coil ' $C$ ', and Strand ' $E$ ') is shown above the sequence. The top line gives the confidence of the predictions, with high bars indicating a more reliable prediction
} 


\section{a}

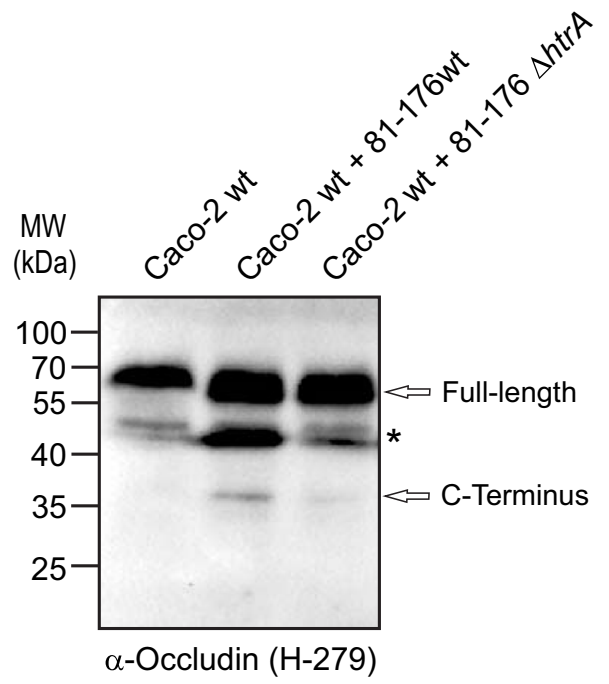

55

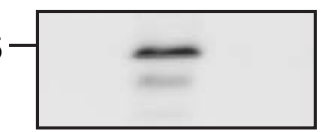

$\alpha-H \operatorname{trA}$

35

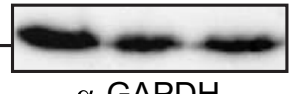

b

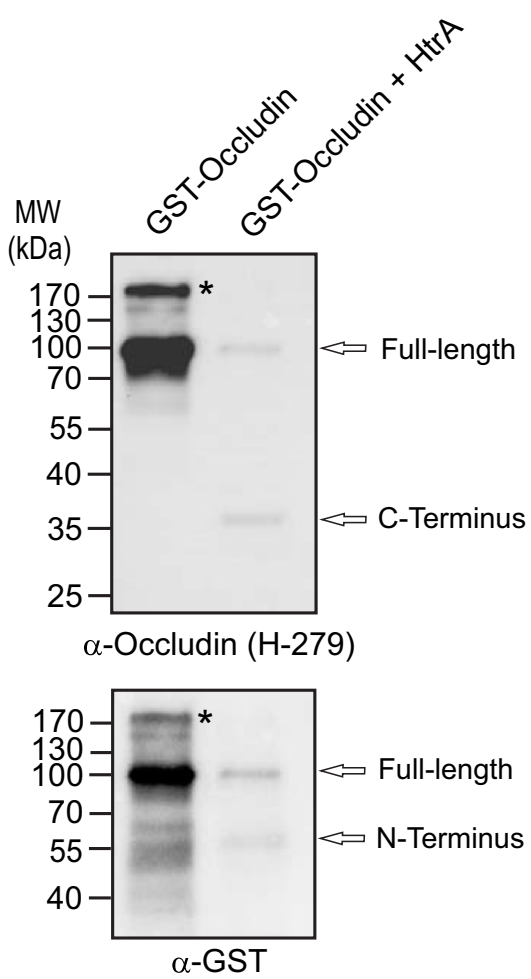

C $100 \mathrm{kDa}$

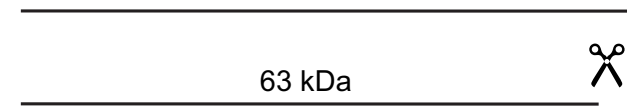

gr

$37 \mathrm{kDa}$

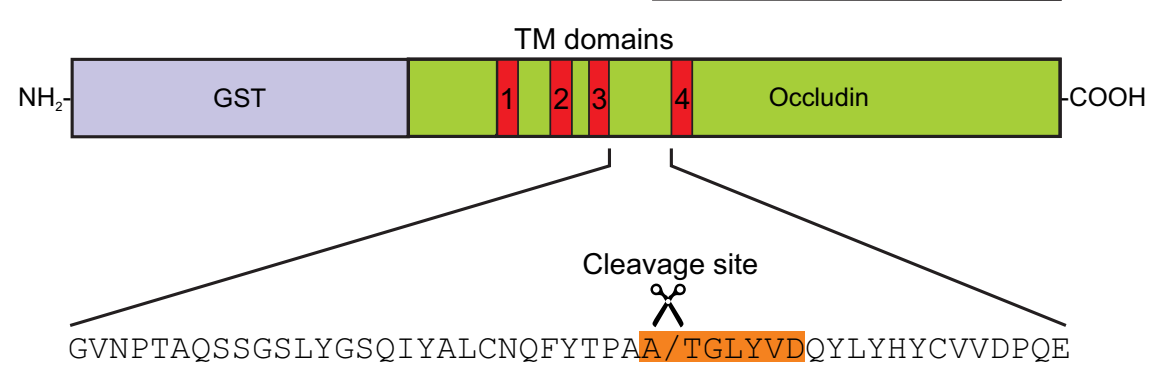

d

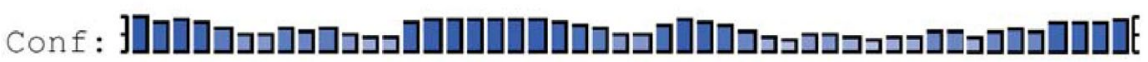

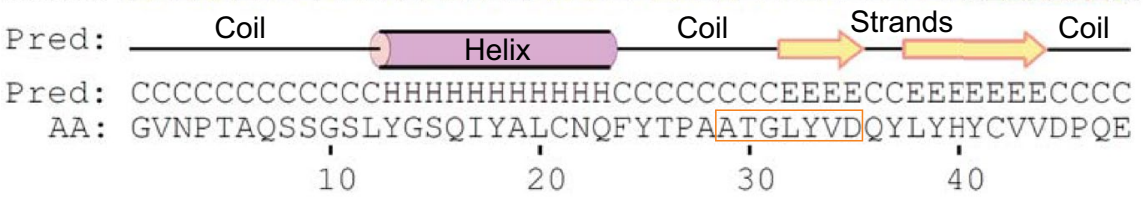

observed occludin dimers of the recombinant protein (Fig. 7b). Interestingly, we noted that HtrA cleavage terminated occludin's dimerization properties. Thus, we propose that HtrA cleavage affects occludin functions by terminating its dimerization and interaction with $\mathrm{ZO}-1$, to increase tight junctional permeability upon infection. 
a Caco-2 wild-type cells

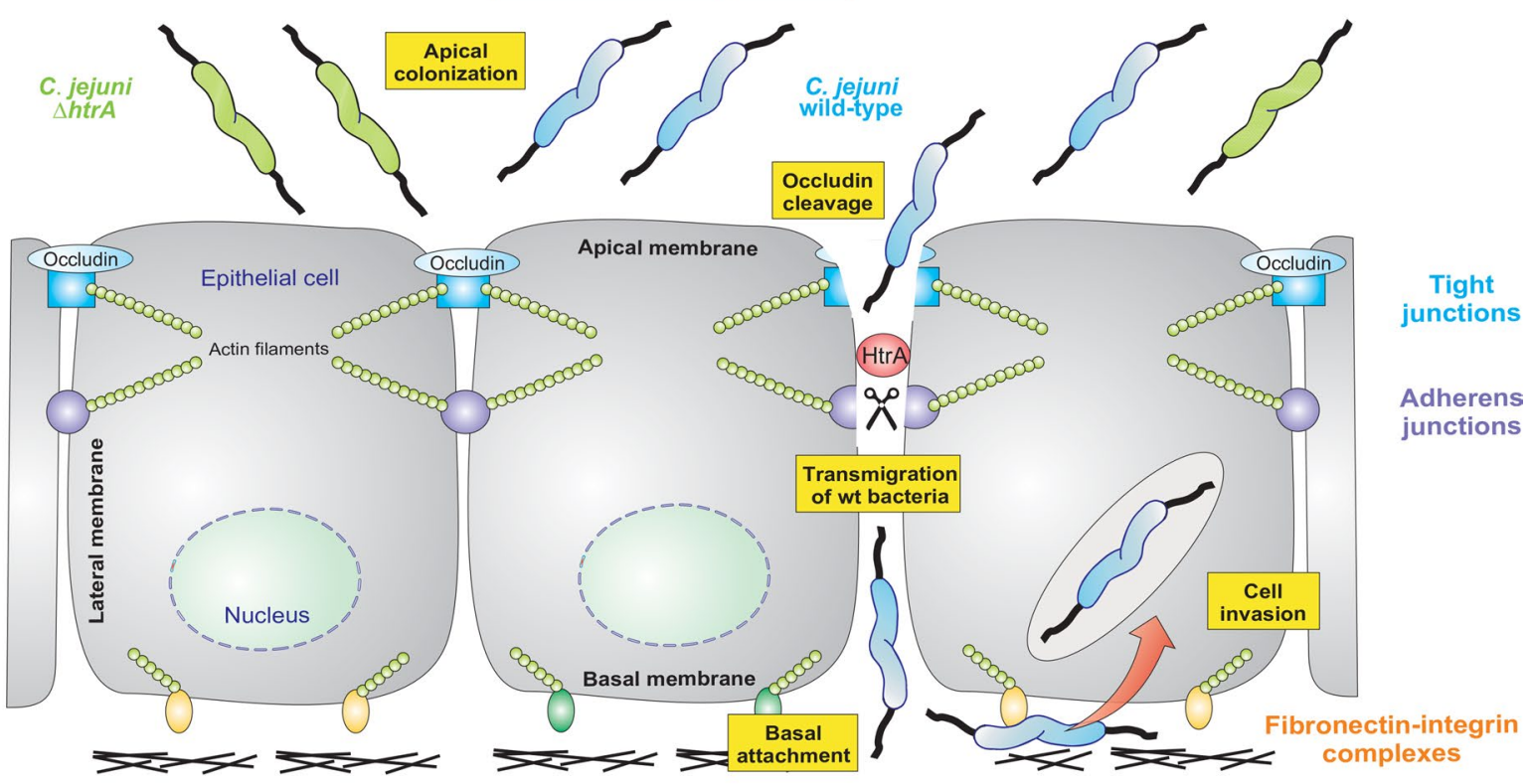

b

\section{Occludin knockout cells}

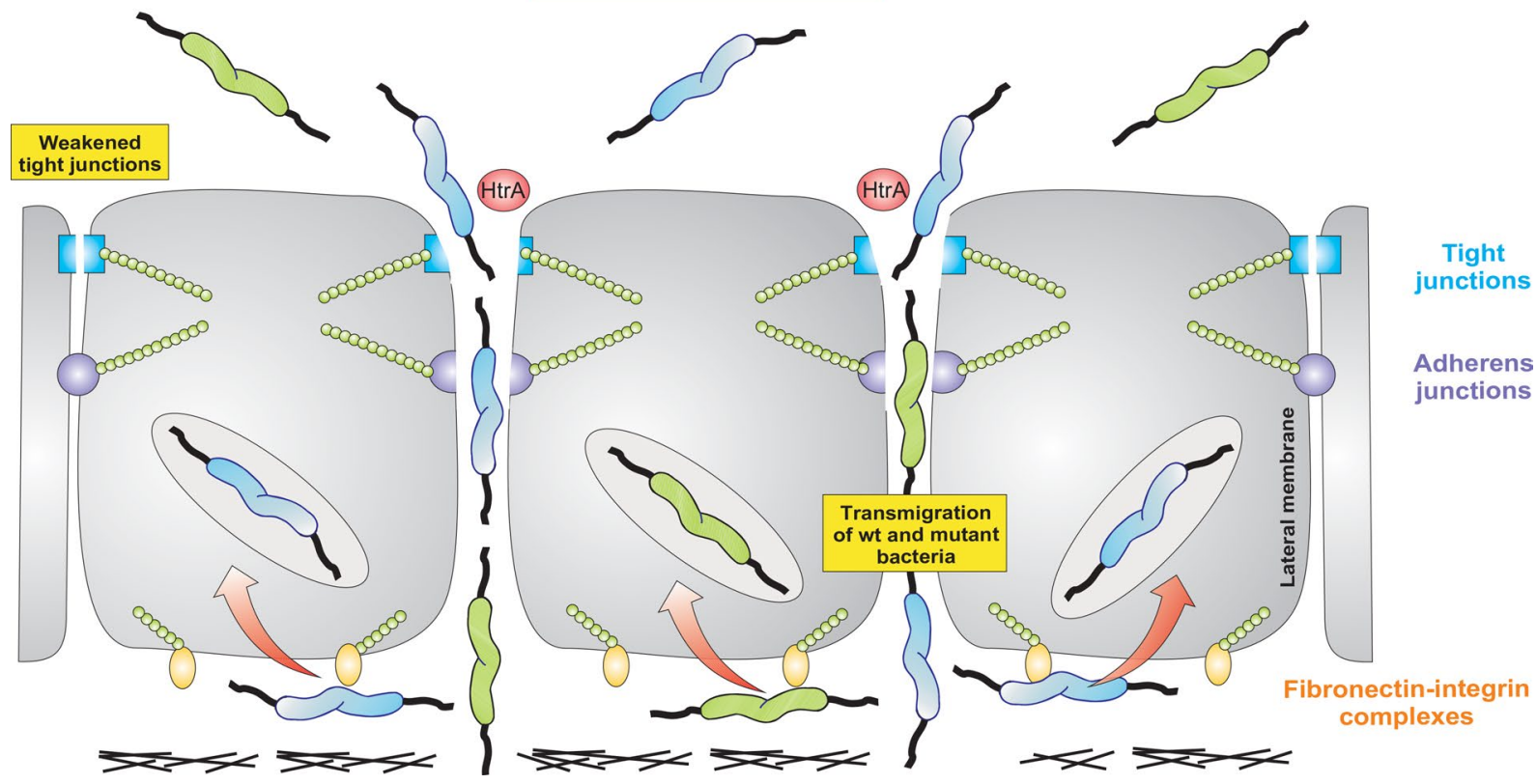

Fig. 8 Model for the transmigration, adhesion and invasion by C. jejuni during infection of polarized Caco-2 cells depends on the expression of HtrA and tight junction protein occludin. a Upon infection of Caco-2 wild-type cells, C. jejuni exploits the secreted serine protease HtrA to cleave the apical tight junction protein occludin (this work) and the adherens junction protein E-cadherin [11]. Cleavage of both factors weakens the tight and adherens junctions, respectively, followed by entering of wild-type C. jejuni into the intercellular space between neighboring cells of the gut epithelium and paracellular transmigration. This enables the bacteria to reach basal surfaces and the fibronectin-integrin complex that connect the cells with underlying tissue. This complex is used as the receptor for the C. jejuni adhesins CadF/FlpA to bind and enter the epithelial cells from the bottom. As cleavage of the junctional proteins by HtrA is required for this outcome, $\triangle$ htrA mutant bacteria are strongly diminished in transmigration, adhesion and invasion of polarized Caco-2 cells. b During infection of Caco-2 cells with occludin knockout, the tight junctions are slightly weaker compared to wild-type cells as confirmed by TER measurement and immunofluorescence microscopy. This leads to enhanced transmigration, adhesion and invasion rates by wild-type C. jejuni. In addition, absence of occludin even allows $\Delta$ trA bacteria to transmigrate to some extent, resulting in increased cell binding and invasion, albeit still at lower rates compared to wild-type C. jejuni 
In line with our hypothesis, both adhesion and invasion of $C$. jejuni strains was increased in occludin knockout cells as compared to Caco-2 wild-type cells. Most likely, the tight junctions of the knockout cells did not provide full protection against the bacterial transmigration, so that more wild-type $C$. jejuni can reach the basal membranes, where they could adhere and invade. Our results suggest that loss of occludin in Caco-2 cells by CRISPR/Cas9 inactivation partially opened this barrier, which phenotypically mimics cleavage of the protein by HtrA, so that wild-type bacteria can better transmigrate between neighbouring cells. This provides a likely mechanism by which secretion of HtrA can assist C. jejuni in reaching the basolateral side of the epithelium and invade deeper tissues. In fact, using the adhesion proteins CadF or FlpA, C. jejuni typically invades epithelial cells through the fibronectin-based focal adhesion complexes at the basolateral membrane, which can only be targeted when the bacteria first penetrate the tight junctions of a confluent cell monolayer [68]. In line with this assumption, the $\Delta h \operatorname{tr} A$ mutant adhered to and invaded into occludin knockout cells at much better rates compared to wild-type Caco-2 cells.

Taken together, our working hypothesis is that $C$. jejuni aims to reach the basal side of polarized epithelial cells by transmigration through the tight and adherens junctions that are at least temporarily opened by the action of HtrA (Fig. 8). Cleavage of occludin may be just one option to open the cell-to-cell junctions. However, occludin is probably not the only target which $C$. jejuni uses to enable transmigration, because HtrA can also cleave the adherens junction protein E-cadherin [11]. In this way, C. jejuni impairs intestinal barrier functions, invades epithelial cells and achieves access to deeper tissues and even other organs. This is an intriguing new mechanism to breach the epithelial barrier of the human host and cause disease. More studies are clearly necessary to investigate this infection strategy and the role of secreted HtrA in more detail.

\section{Authors' contributions}

AH performed the studies of Caco-2 cell infections, RB performed the immunofluorescence studies on human biopsies, MB designed the gentamicin protection and transwell assays, UZ purified recombinant HtrA, NT did the in vitro cleavage experiments, HS did the bioinformatics analysis, JDS analyzed the data, SB conceptualized the study, analyzed the data and wrote the paper. All authors read and approved the final manuscript.

\section{Author details}

1 Division of Microbiology, Dept. of Biology, University of Erlangen-Nuremberg, Staudtstr. 5, 91058 Erlangen, Germany. ${ }^{2}$ Institut für Klinische Physiologie, Med. Klinik m.S. Gastroenterologie, Infektiologie und Rheumatologie, Charité-Universitätsmedizin Berlin, Berlin, Germany. ${ }^{3}$ Division of Bioinformatics, Institute of Biochemistry, Friedrich-Alexander-Universität Erlangen-Nürnberg, Erlangen, Germany. ${ }^{4}$ Department of General and Medical Biochemistry, Faculty of Biology, University of Gdansk, 80-308 Gdansk, Poland.

\section{Acknowledgements}

We thank Dr. Christof Hauck (University of Konstanz) for his support with generation of the occludin CRISPR-Cas9 knockout cells.

\section{Competing interests}

The authors declare that they have no competing interests.

\section{Availability of data and materials}

The data that support the findings of this study are available from the corresponding author upon request.

\section{Consent for publication}

Not applicable.

\section{Ethics approval and consent of participate}

For the use of human gut biopsy material, this study adhered to the Declaration of Helsinki. The study was approved by the Ethics Committee of the Charité (Berlin, Germany) under Approval Number EA4/123/09 and written informed consent was obtained from all sampled individuals.

\section{Funding}

This work is supported by German Federal Ministries of Education and Research (BMBF) in frame of the zoonoses research consortium PAC-Campylobacter to SB (IP9/01Kl1725E) and RB \& JDS (IP8/01KI1725D).

\section{Publisher's Note}

Springer Nature remains neutral with regard to jurisdictional claims in published maps and institutional affiliations.

Received: 30 October 2018 Accepted: 1 February 2019

Published online: 13 February 2019

\section{References}

1. Ketley JM. Pathogenesis of enteric infection by Campylobacter. Microbiology (Reading, England). 1997;143(Pt 1):5-21.

2. Wooldridge KG, Ketley JM. Campylobacter-host cell interactions. Trends Microbiol. 1997:5:96-102.

3. Young KT, Davis LM, Dirita VJ. Campylobacter jejuni: molecular biology and pathogenesis. Nat Rev Microbiol. 2007;5:665-79.

4. van Spreeuwel JP, Duursma GC, Meijer CJ, Bax R, Rosekrans PC, Lindeman J. Campylobacter colitis: histological immunohistochemical and ultrastructural findings. Gut. 1985;26:945-51.

5. Moser I, Schroeder W, Salnikow J. Campylobacter jejuni major outer membrane protein and a 59-kDa protein are involved in binding to fibronectin and INT 407 cell membranes. FEMS Microbiol Lett. 1997;157:233-8.

6. Konkel ME, Monteville MR, Rivera-Amill V, Joens LA. The pathogenesis of Campylobacter jejuni-mediated enteritis. Curr Issues Intest Microbiol. 2001;2:55-71.

7. Krause-Gruszczynska M, Boehm M, Rohde M, Tegtmeyer N, Takahashi S, Buday $L$, et al. The signaling pathway of Campylobacter jejuni-induced Cdc42 activation: role of fibronectin, integrin beta1, tyrosine kinases and guanine exchange factor Vav2. Cell Commun Signal. 2011;9:32.

8. Boehm M, Krause-Gruszczynska M, Rohde M, Tegtmeyer N, Takahashi S, Oyarzabal OA, et al. Major host factors involved in epithelial cell invasion of Campylobacter jejuni: role of fibronectin, integrin beta1, FAK, Tiam-1, and DOCK180 in activating Rho GTPase Rac1. Front Cell Infection Microbiol. 2011:1:17.

9. Eucker TP, Konkel ME. The cooperative action of bacterial fibronectinbinding proteins and secreted proteins promote maximal Campylobacter jejuni invasion of host cells by stimulating membrane ruffling. Cell Microbiol. 2012;14:226-38.

10. Backert S, Boehm M, Wessler S, Tegtmeyer N. Transmigration route of Campylobacter jejuni across polarized intestinal epithelial cells: paracellular, transcellular or both? Cell Commun Signal. 2013;11:72.

11. Boehm M, Hoy B, Rohde M, Tegtmeyer N, Baek KT, Oyarzabal OA, et al. Rapid paracellular transmigration of Campylobacter jejuni across polarized epithelial cells without affecting TER: role of proteolytic-active HtrA cleaving E-cadherin but not fibronectin. Gut Pathog. 2012;4:3. 
12. Cortes G, de Astorza B, Benedi VJ, Alberti S. Role of the htrA gene in Klebsiella pneumoniae virulence. Infect Immun. 2002;70:4772-6.

13. Mo E, Peters SE, Willers C, Maskell DJ, Charles IG. Single, double and triple mutants of Salmonella enterica serovar Typhimurium degP (htrA), degQ (hhoA) and $\operatorname{deg} S$ (hhoB) have diverse phenotypes on exposure to elevated temperature and their growth in vivo is attenuated to different extents. Microb Pathog. 2006;41:174-82.

14. Li SR, Dorrell N, Everest PH, Dougan G, Wren BW. Construction and characterization of a Yersinia enterocolitica 0:8 high-temperature requirement (htrA) isogenic mutant. Infect Immun. 1996;64:2088-94.

15. Humphreys S, Stevenson A, Bacon A, Weinhardt AB, Roberts M. The alternative sigma factor, sigmaE, is critically important for the virulence of Salmonella typhimurium. Infect Immun. 1999;67:1560-8.

16. Purdy GE, Hong M, Payne SM. Shigella flexneri DegP facilitates IcsA surface expression and is required for efficient intercellular spread. Infect Immun. 2002;70:6355-64

17. Ye M, Sharma K, Thakur M, Smith AA, Buyuktanir O, Xiang X, et al. HtrA, a temperature- and stationary phase-activated protease involved in maturation of a key microbial virulence determinant, facilitates Borrelia burgdorferi infection in mammalian hosts. Infect Immun. 2016:84:2372-81.

18. Wilson RL, Brown LL, Kirkwood-Watts D, Warren TK, Lund SA, King DS, et al. Listeria monocytogenes $10403 \mathrm{~S} \mathrm{HtrA}$ is necessary for resistance to cellular stress and virulence. Infect Immun. 2006;74:765-8.

19. Kim DY, Kim KK. Structure and function of HtrA family proteins, the key players in protein quality control. J Biochem Mol Biol. 2005;38:266-74.

20. Skorko-Glonek J, Zurawa-Janicka D, Koper T, Jarzab M, Figaj D, Glaza $P$, et al. HtrA protease family as therapeutic targets. Curr Pharm Des. 2013;19:977-1009.

21. Lipinska B, Fayet O, Baird L, Georgopoulos C. Identification, characterization, and mapping of the Escherichia coli htrA gene, whose product is essential for bacterial growth only at elevated temperatures. J Bacteriol. 1989;171:1574-84.

22. Clausen T, Kaiser M, Huber R, Ehrmann M. HTRA proteases: regulated proteolysis in protein quality control. Nat Rev Mol Cell Biol. 2011;12:152-62.

23. Hoy B, Geppert T, Boehm M, Reisen F, Plattner P, Gadermaier G, et al. Distinct roles of secreted HtrA proteases from Gram-negative pathogens in cleaving the junctional protein and tumor suppressor E-cadherin. J Biol Chem. 2012;287:10115-20.

24. Turner JR. Molecular basis of epithelial barrier regulation: from basic mechanisms to clinical application. Am J Pathol. 2006;169:1901-9.

25. Gunzel D, Yu AS. Claudins and the modulation of tight junction permeability. Physiol Rev. 2013;93:525-69.

26. Guttman JA, Finlay BB. Tight junctions as targets of infectious agents. Biochem Biophys Acta. 2009;1788:832-41.

27. Van Itallie CM, Anderson JM. Architecture of tight junctions and principles of molecular composition. Semin Cell Dev Biol. 2014;36:157-65.

28. Feldman GJ, Mullin JM, Ryan MP. Occludin: structure, function and regulation. Adv Drug Deliv Rev. 2005;57:883-917.

29. Wong V. Phosphorylation of occludin correlates with occludin localization and function at the tight junction. Am J Physiol. 1997;273:C1859-67.

30. Dorfel MJ, Westphal JK, Bellmann C, Krug SM, Cording J, Mittag S, et al. CK2-dependent phosphorylation of occludin regulates the interaction with ZO-proteins and tight junction integrity. Cell Commun Signal. 2013;11:40.

31. Raleigh DR, Boe DM, Yu D, Weber CR, Marchiando AM, Bradford EM, et al. Occludin $\mathrm{S} 408$ phosphorylation regulates tight junction protein interactions and barrier function. J Cell Biol. 2011;193:565-82.

32. Furuse $M$, Itoh M, Hirase T, Nagafuchi A, Yonemura S, Tsukita S, et al. Direct association of occludin with ZO-1 and its possible involvement in the localization of occludin at tight junctions. J Cell Biol. 1994;127:1617-26.

33. Ronaghan NJ, Shang J, lablokov V, Zaheer R, Colarusso P, Dion S, et al. The serine protease-mediated increase in intestinal epithelial barrier function is dependent on occludin and requires an intact tight junction. Am J Physiol Gastrointest Liver Physiol. 2016;311:G466-79.

34. Alemka A, Clyne M, Shanahan F, Tompkins T, Corcionivoschi N, Bourke B. Probiotic colonization of the adherent mucus layer of HT29MTXE12 cells attenuates Campylobacter jejuni virulence properties. Infect Immun 2010;78:2812-22

35. MacCallum A, Hardy SP, Everest PH. Campylobacter jejuni inhibits the absorptive transport functions of Caco-2 cells and disrupts cellular tight junctions. Microbiology (Reading, England). 2005;151:2451-8.
36. Chen ML, Ge Z, Fox JG, Schauer DB. Disruption of tight junctions and induction of proinflammatory cytokine responses in colonic epithelial cells by Campylobacter jejuni. Infect Immun. 2006;74:6581-9.

37. Elmi A, Dorey A, Watson E, Jagatia H, Inglis NF, Gundogdu O, et al. The bile salt sodium taurocholate induces Campylobacter jejuni outer membrane vesicle production and increases OMV-associated proteolytic activity. Cell Microbiol. 2018. https://doi.org/10.1111/cmi.12814.

38. Elmi A, Nasher F, Jagatia H, Gundogdu O, Bajaj-Elliott M, Wren B, et al. Campylobacter jejuni outer membrane vesicle-associated proteolytic activity promotes bacterial invasion by mediating cleavage of intestinal epithelial cell E-cadherin and occludin. Cell Microbiol. 2016;18:561-72.

39. Boehm M, Lind J, Backert S, Tegtmeyer N. Campylobacter jejuni serine protease HtrA plays an important role in heat tolerance, oxygen resistance, host cell adhesion, invasion, and transmigration. Eur J Microbiol Immunol. 2015;5:68-80

40. Krause-Gruszczynska M, van Alphen LB, Oyarzabal OA, Alter T, Hanel I, Schliephake A, et al. Expression patterns and role of the CadF protein in Campylobacter jejuni and Campylobacter coli. FEMS Microbiol Lett. 2007;274:9-16

41. Bucker R, Krug SM, Moos V, Bojarski C, Schweiger MR, Kerick M, et al Campylobacter jejuni impairs sodium transport and epithelial barrier function via cytokine release in human colon. Mucosal Immunol. 2018;11:474-85.

42. Zarzecka U, Modrak-Wojcik A, Bayassi M, Szewczyk M, Gieldon A, Lesner A, et al. Biochemical properties of the HtrA homolog from bacterium Stenotrophomonas maltophilia. Int J Biol Macromol. 2018;109:992-1005.

43. Hartung ML, Gruber DC, Koch KN, Gruter L, Rehrauer H, Tegtmeyer N, et al. H. pylori-induced DNA strand breaks are introduced by nucleotide excision repair endonucleases and promote NF-kappaB target gene expression. Cell Rep. 2015;13:70-9.

44. Brondsted L, Andersen MT, Parker M, Jorgensen $\mathrm{K}$, Ingmer $\mathrm{H}$. The HtrA protease of Campylobacter jejuni is required for heat and oxygen tolerance and for optimal interaction with human epithelial cells. Appl Environ Microbiol. 2005;71:3205-12.

45. Hirsch C, Tegtmeyer N, Rohde M, Rowland M, Oyarzabal OA, Backert S. Live Helicobacter pylori in the root canal of endodontic-infected decidu ous teeth. J Gastroenterol. 2012;47:936-40.

46. KwokT, Backert S, Schwarz H, Berger J, Meyer TF. Specific entry of Helicobacter pylori into cultured gastric epithelial cells via a zipper-like mechanism. Infect Immun. 2002;70:2108-20.

47. Krause-Gruszczynska M, Rohde M, Hartig R, Genth H, Schmidt G, Keo T, et al. Role of the small Rho GTPases Rac1 and Cdc42 in host cell invasion of Campylobacter jejuni. Cell Microbiol. 2007;9:2431-44.

48. Brisslert M, Enarsson K, Lundin S, Karlsson A, Kusters JG, Svennerholm AM, Backert S, Quiding-Järbrink M. Helicobacter pylori induce neutrophil transendothelial migration: role of the bacterial HP-NAP. FEMS Microbiol Lett. 2005:249:95-103.

49. Buchan DW, Minneci F, Nugent TC, Bryson K, Jones DT. Scalable web services for the PSIPRED protein analysis workbench. Nucleic Acids Res. 2013;41:W349-57.

50. Schmidt TP, Perna AM, Fugmann T, Bohm M, Jan H, Haller S, et al. Identification of E-cadherin signature motifs functioning as cleavage sites for Helicobacter pylori HtrA. Sci Rep. 2016;6:23264.

51. Landau D. Epithelial paracellular proteins in health and disease. Curr Opin Nephrol Hypertens. 2006;15:425-9.

52. Vogelmann R, Amieva MR, Falkow S, Nelson WJ. Breaking into the epithelial apical-junctional complex-news from pathogen hackers. Curr Opin Cell Biol. 2004;16:86-93.

53. Eichner M, Augustin C, Fromm A, Piontek A, Walther W, Bucker R, et al. In colon epithelia, Clostridium perfringens enterotoxin causes focal leaks by targeting claudins which are apically accessible due to tight junction derangement. J Infect Dis. 2017;217:147-57.

54. Wu Z, Nybom P, Magnusson KE. Distinct effects of Vibrio cholerae haemagglutinin/protease on the structure and localization of the tight junction-associated proteins occludin and ZO-1. Cell Microbiol. 2000;2:11-7.

55. Tegtmeyer N, Wessler S, Necchi V, Rohde M, Harrer A, Rau TT, et al. Helicobacter pylori employs a unique basolateral type IV secretion mechanism for CagA delivery. Cell Host Microbe. 2017;22(552-60):e5.

56. Wroblewski LE, Shen L, Ogden S, Romero-Gallo J, Lapierre LA, Israel DA, et al. Helicobacter pylori dysregulation of gastric epithelial tight 
junctions by urease-mediated myosin II activation. Gastroenterology. 2009;136:236-46.

57. Bao J, Yura RE, Matters GL, Bradley SG, Shi P, Tian F, Bond JS. Meprin A impairs epithelial barrier function, enhances monocyte migration, and cleaves the tight junction protein occludin. Am J Physiol Renal Physiol. 2013;305:F714-26.

58. Ulitzky L, Lafer MM, KuKuruga MA, Silberstein E, Cehan N, Taylor DR. A new signaling pathway for HCV inhibition by estrogen: GPR30 activation leads to cleavage of occludin by MMP-9. PLoS ONE. 2016;11:e0145212.

59. Saitou M, Furuse M, Sasaki H, Schulzke JD, Fromm M, Takano H, et al. Complex phenotype of mice lacking occludin, a component of tight junction strands. Mol Biol Cell. 2000;1 1:4131-42.

60. Schulzke JD, Gitter AH, Mankertz J, Spiegel S, Seidler U, Amasheh S, et al. Epithelial transport and barrier function in occludin-deficient mice. Biochem Biophys Acta. 2005;1669:34-42.

61. Ikenouchi J, Sasaki H, Tsukita S, Furuse M, Tsukita S. Loss of occludin affects tricellular localization of tricellulin. Mol Biol Cell. 2008;19:4687-93.

62. Nusrat A, Chen JA, Foley CS, Liand TW, Tom J, Cromwell M, Quan C, Mrsny RJ. The coiled-coil domain of occludin can act to organize structural and functional elements of the epithelial tight junction. J Biol Chem. 2000;275:29816-22.

63. Blasig IE, Winkler L, Lassowski B, Mueller SL, Zuleger N, Krause E, Krause G, Gast K, Kolbe M, Piontek J. On the self-association potential of transmembrane tight junction proteins. Cell Mol Life Sci. 2006;63:505-14.
64. Muller SL, Portwich M, Schmidt A, Utepbergenov DI, Huber O, Blasig IE, Krause $\mathrm{G}$. The tight junction protein occludin and the adherens junction protein alpha-catenin share a common interaction mechanism with ZO-1. J Biol Chem. 2005;280:3747-56.

65. Balda MS, Whitney JA, Flores C, Gonzalez S, Cereijido M, Matter K. Functional dissociation of paracellular permeability and transepithelial electrical resistance and disruption of the apical-basolateral intramembrane diffusion barrier by expression of a mutant tight junction membrane protein. J Cell Biol. 1996;134:1031-49.

66. Chen YH, Merzdorf C, Paul DL, Goodenough DA. C-terminus of occludin is required for tight junction barrier function in early Xenopus embryos. J Cell Biol. 1997:138:891-9.

67. Walter JK, Castro V, Voss M, Gast K, Rueckert C, Piontek J, Blasig IE. Redox-sensitivity of the dimerization of occludin. Cell Mol Life Sci. 2009;66:3655-62.

68. O Cróinín T, Backert S: Host epithelial cell invasion by Campylobacter jejuni: trigger or zipper mechanism? Frontiers in cellular and infection microbiology 2012, 2:25.

69. Baek KT, Vegge CS, Skorko-Glonek J, Brondsted L. Different contributions of $\mathrm{HtrA}$ protease and chaperone activities to Campylobacter jejuni stress tolerance and physiology. Appl Environ Microbiol. 2011;77:57-66.
Ready to submit your research? Choose BMC and benefit from:

- fast, convenient online submission

- thorough peer review by experienced researchers in your field

- rapid publication on acceptance

- support for research data, including large and complex data types

- gold Open Access which fosters wider collaboration and increased citations

- maximum visibility for your research: over 100M website views per year

At BMC, research is always in progress.

Learn more biomedcentral.com/submissions 